\title{
Bioinformatics for the NuGO proof of principle study: analysis of gene expression in muscle of ApoE3*Leiden mice on a high-fat diet using PathVisio
}

\author{
Susan L. M. Coort · Martijn P. van Iersel · \\ Marjan van Erk · Teake Kooistra • \\ Robert Kleemann · Chris T. A. Evelo
}

Received: 8 October 2008/Accepted: 12 November 2008/Published online: 26 November 2008

(c) The Author(s) 2008. This article is published with open access at Springerlink.com

\begin{abstract}
Insulin resistance is a characteristic of type-2 diabetes and its development is associated with an increased fat consumption. Muscle is one of the tissues that becomes insulin resistant after high fat (HF) feeding. The aim of the present study is to identify processes involved in the development of HF-induced insulin resistance in muscle of ApOE $3 *$ Leiden mice by using microarrays. These mice are known to become insulin resistant on a HF diet. Differential gene expression was measured in muscle using the Affymetrix mouse plus 2.0 array. To get more insight in the processes, affected pathway analysis was performed with a new tool, PathVisio. PathVisio is a pathway editor customized with plug-ins (1) to visualize microarray data on pathways and (2) to perform statistical analysis to select pathways of interest. The present study demonstrated that with pathway analysis, using PathVisio, a large variety of processes can be investigated. The significantly regulated genes in muscle of ApOE3*Leiden mice after 12 weeks of HF feeding were involved in several biological pathways including fatty acid beta oxidation, fatty acid biosynthesis, insulin signaling, oxidative stress and inflammation.
\end{abstract}

Keywords Pathway analysis - Microarray ·

Insulin resistance $\cdot$ High-fat diet

S. L. M. Coort $(\square)$ - M. P. van Iersel · C. T. A. Evelo Department of Bioinformatics-BiGCaT, Maastricht University, P.O. Box 616, 6200 MD Maastricht, The Netherlands e-mail: susan.coort@bigcat.unimaas.nl

M. van Erk

TNO Quality of Life, Zeist, The Netherlands

T. Kooistra $\cdot$ R. Kleemann

TNO Quality of Life, Leiden, The Netherlands

\section{Introduction}

The prevalence of type-2 diabetes (T2D) is increasing dramatically worldwide. One of the characteristics of T2D is insulin resistance (IR). IR is a reduced responsiveness of cells to the action of insulin. It has been demonstrated that the consumption of a high fat (HF) diet is positively correlated with the development of IR in humans and rodents [7]. Under physiological conditions, insulin stimulates the cellular uptake and metabolism of glucose in muscle and other tissues [15]. Muscle has an important role in the maintenance of the glucose homeostasis. It is known that a HF-diet can influence glucose metabolism in muscle [4]. Therefore, it is of interest to investigate which biological processes are regulated in HF diet-induced insulin resistance in muscle. A well-described animal model that becomes insulin resistant after feeding a HF diet is the ApOE3*Leiden mouse model [12]. ApOE3*Leiden mice express the human ApOE $3 *$ Leiden gene which results in a lipoprotein profile that closely resembles that of humans [19]. The aim of the present study is to identify processes involved in the development of high-fat induced insulin resistance in muscle of ApOE3*Leiden mice by using microarrays.

Microarray analysis has enabled the measurement of thousands of genes in a single RNA sample. This results in the generation of an enormous amount of gene expression data. Lists of significantly regulated genes are generated based on this data. However, when looking only at lists of genes, no information is provided about the processes in which they are involved. A manner to examine biological processes is by performing pathway analysis [1]. In this study, the pathway analysis was performed using the PathVisio pathway editor [17], customized with plug-ins (1) to visualize microarray data on pathways and (2) to perform statistical analysis to select pathways of interest. 


\section{Methods}

\section{Animal model and RNA extraction}

APOE*3 Leiden transgenic mice were fed a high fat $(\mathrm{HF})$ diet for 12 weeks. As a control group $(t=0)$ chow-fed APOE*3Leiden mice were used. The control and treatment group consisted of 7 and 8 animals, respectively. Muscle tissue was collected in both groups and freeze-clamped immediately after harvesting. Total RNA for microarray analysis was extracted from individual tissues and the integrity of each RNA sample obtained was examined as previously described [9]. RNA was labeled and hybridized to Affymetrix MOE430-2.0 arrays.

\section{Microarray data analysis}

Differential gene expression was measured using the Affymetrix MOE430-2.0 arrays. Quality control was performed using the $\mathrm{R}$ pipeline which can be accessed from Madmax (see https://madmax.bioinformatics.nl) or from Genepattern [2]. All 15 arrays passed the quality control and were used for normalization. The normalization method used was GCRMA slow, which uses GC content of the probes in Robust Multiarray Analysis. The arrays were annotated using custom CDF v9.0.1 resulting in the reannotation of the probe sets into Entrez gene-ids. Filtering was performed for low expressed genes. Genes were selected with an expression $>5$ in at least four samples. In muscle 10,777 genes met this filtering criterion and were used for further statistical analysis.

\section{Statistical analysis}

Statistical analysis was performed on a NuGO blackbox (see http://nbx2.nugo.org) using the limma R-package [16] which is part of bioconductor (http://www.bioconductor. org). Within the limma package Empirical Bayes statistics [3] was performed comparing $t=12$ with $t=0$ and resulting in a fold change, $P$ value and false discovery rate.

Pathway analysis

PathVisio 1.1 was used to perform pathway analysis. Custom plug-ins for visualizing microarray data and calculating $z$ scores were added to PathVisio. The mouse
Table 1 The 25 highest ranked pathways based on $z$ score

Genes were considered to be regulated (positive for the set criterion) if the fold change (FC) is smaller than -1.2 or higher than 1.2 and the $P$ value $\leq 0.05$. Positive $(r)$ are the number of genes in the pathway that meet the above-mentioned criterion. Measured ( $n$ ) are the number of genes in the pathway that are present on the microarray. Total is the total amount of genes in a specific pathway. Percentage (\%) is the percentage of the measured genes meeting the set criterion

\begin{tabular}{lrrrrl}
\hline Pathway & Positive $(r)$ & Measured $(n)$ & Total & $\%$ & $z$ Score \\
\hline Fatty acid biosynthesis & \multicolumn{1}{c}{ 15 } & 19 & 22 & 78.95 & 5.91 \\
Fatty acid beta oxidation 1 & 14 & 23 & 27 & 60.87 & 4.42 \\
Muscle, fat and connective tissue specific genes & 16 & 28 & 55 & 57.14 & 4.41 \\
Pentose phosphate pathway & 6 & 7 & 8 & 85.71 & 4.01 \\
Beta oxidation meta MAPP & 15 & 28 & 32 & 53.57 & 3.95 \\
Mitochondrial LC-fatty acid beta-oxidation & 9 & 15 & 16 & 60.00 & 3.49 \\
Genes specific to internal organs & 30 & 79 & 202 & 37.97 & 3.32 \\
Genes specific to blood and lymph tissue & 7 & 11 & 17 & 63.64 & 3.28 \\
Glutathione metabolism & 9 & 16 & 38 & 56.25 & 3.24 \\
Irinotecan pathway & 5 & 7 & 13 & 71.43 & 3.11 \\
Fatty acid beta oxidation 2 & 4 & 6 & 6 & 66.67 & 2.6 \\
Genes specific to blood and lymph tissue & 9 & 21 & 40 & 42.86 & 2.24 \\
Myometrial relaxation and contraction pathways & 33 & 105 & 161 & 31.43 & 2.22 \\
Alanine and aspartate metabolism & 4 & 7 & 40 & 57.14 & 2.2 \\
Inflammatory response pathway & 10 & 25 & 41 & 40.00 & 2.11 \\
Cholesterol biosynthesis & 5 & 10 & 15 & 50.00 & 2.09 \\
Circadian exercise & 13 & 35 & 49 & 37.14 & 2.09 \\
Triacylglyceride synthesis & 8 & 19 & 25 & 42.11 & 2.06 \\
Mouse insulin signaling & 36 & 121 & 160 & 29.75 & 1.94 \\
Oxidative stress & 9 & 23 & 29 & 39.13 & 1.92 \\
G Protein signaling pathways & 20 & 61 & 87 & 32.79 & 1.89 \\
Tryptophan metabolism & 11 & 30 & 87 & 36.67 & 1.87 \\
Fatty acid beta oxidation 3 & 4 & 8 & 50.00 & 1.87 \\
Striated muscle contraction & 21 & 45 & 38.10 & 1.72 \\
Nuclear receptors in lipid metabolism and toxicity & 7 & 18 & 34 & 38.89 & 1.67 \\
\hline & & & &
\end{tabular}


gene database of January 2008 and the mouse pathways available on August 2008 were used. Genes were considered to be regulated when meeting the following criterion: average fold change (FC) $\leq-1.2$ or $\geq 1.2$ and $P$ value $\leq 0.05$. Per pathway a $z$ score was calculated by subtracting the expected number of genes meeting the above-mentioned criterion from the observed number and then dividing by the standard deviation of the observed number of genes. A positive $z$ score indicates that more genes met the criterion than would be expected by chance. Thus, the pathways with a positive $z$ score were considered to be of interest.

\section{Results}

From the 10,776 genes that passed the quality control, 10,445 genes could be linked to Ensembl identifiers by PathVisio. Only 2,090, i.e. $\sim 20 \%$, of these genes were associated with the mouse pathways in the PathVisio repository. Based on these 2,090 genes, pathways of interest were selected. Per pathway a $z$ score was calculated (see "Methods" for a detailed description) and the pathways were ranked based on the $z$ score. The pathways with the highest positive $z$ score were ranked as most regulated.
The 25 highest ranked pathways are listed in Table 1. In the top 25 many pathways related to fatty acid metabolism are present. Moreover, the insulin-signaling pathway is ranked high. The fatty-acid oxidation, fatty acid biosynthesis and insulin-signaling pathways will be discussed in more detail below.

Fatty acid metabolism

Most genes present in the Mus musculus fatty acid beta oxidation 1 pathway (see Fig. 1 and http://wikipathways. org) are significantly downregulated after 12 weeks of HF feeding in muscle of ApoE3*Leiden mice. The expression of hormone sensitive lipase ( -5.39 -fold), lipoprotein lipase (-2.04-fold) and mitochondrial short chain enoyl Coenzyme A hydratase ( -1.58 -fold) is decreased compared to the control group $(t=0)$. Not only the oxidation of fatty acids but also the fatty acid biosynthesis is downregulated. In the Mus musculus fatty acid biosynthesis pathway (see Fig. 2 and http://wikipathways.org), ATP citrate lyase ( -10.37 -fold), fatty acid synthase (-26.21-fold) and stearoyl-Coenzyme A desaturase 1 (-2.43-fold) are among the genes of which the expression is significantly decreased after 12 weeks of HF feeding.

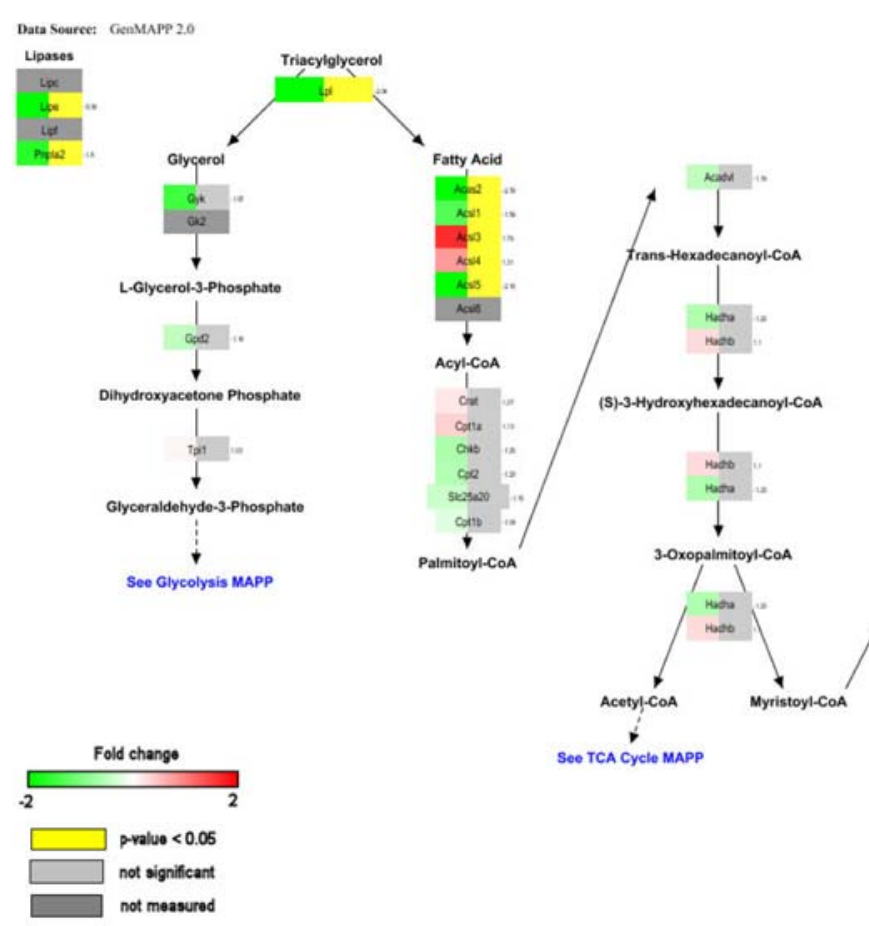

Fig. 1 Gene expression data mapped on the Mus musculus fatty acid beta oxidation 1 pathway. Fold changes are represented as a gradient and visualized per genebox on the left. Yellow boxes indicate a significance of $P \leq 0.05$ and are visualized per genebox on the right.

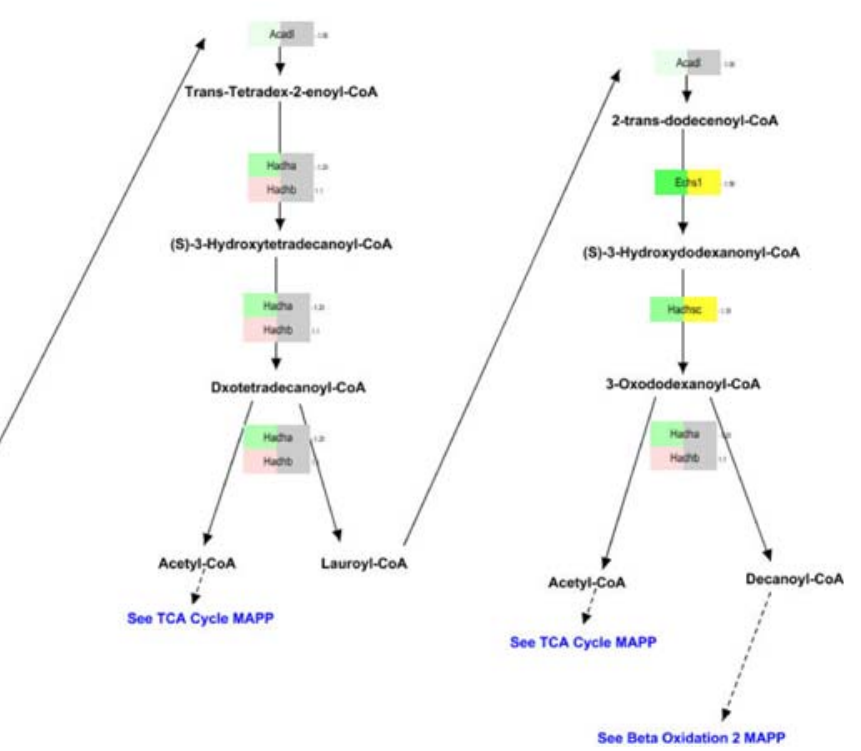

Light gray boxes indicate not significant and are also visualized on the right. The dark gray boxes represent the genes that could not be measured 


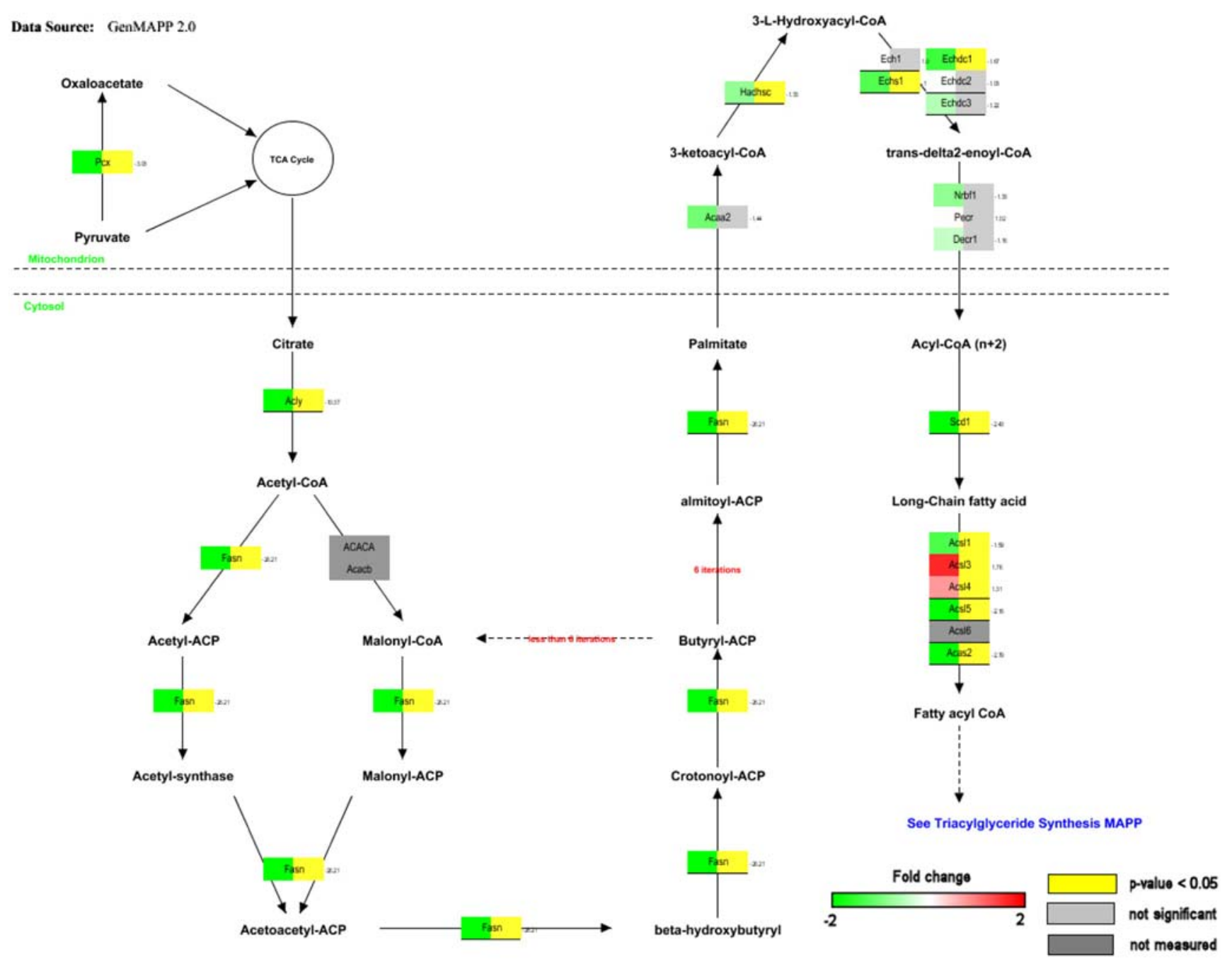

Fig. 2 Gene expression data mapped on the Mus musculus fatty acid synthesis pathway. Fold changes are represented as a gradient and visualized per genebox on the left. Yellow boxes indicate a significance of $P \leq 0.05$ and are visualized per genebox on the right. Light

\section{Insulin signaling}

The mouse insulin signaling pathway is a very extensive pathway (see Fig. 3 and http://wikpathways.org). This pathway is divided into several parts like (1) vesicular transport, (2) PKB/Akt signaling and (3) metabolic regulation, which are important processes in the cellular insulin response. The present results demonstrate that the expression of the main insulin sensitive glucose transporter $\quad$ GLUT4, genename $=$ Slc2a $4, \quad-1.23$-fold $)$ is significantly decreased in muscle after 12 weeks. In contrast, the expression of GLUT1 (Slc2a1) remains unchanged. The PKB/Akt signaling appears to be slightly upregulated reflected by an increased expression of 3-phosphoinositide dependent protein kinase-1 (PDK1, 1.15 -fold), glycogen synthase kinase 3 beta (GSK3b, gray boxes indicate not significant and are also visualized on the right. The dark gray boxes represent the genes that could not be measured

1.15-fold) and glucocorticoid regulated kinase (GSK, 1.22 -fold). In addition, the key genes involved in the vesicular transport of GLUT4 are significantly upregulated, i.e. Ras-related protein Rab-4A (1.7-fold), syntaxin binding protein $3 \mathrm{~A}$ (1.48-fold), syntaxin binding protein 4 (1.33-fold) and vesicle-associated membrane protein 2 (Vamp2, 1.45-fold). Finally, the expression of genes belonging to the metabolic regulation of insulin, i.e. phosphofructokinase $(-1.99$-fold $)$, hormone sensitive lipase ( -5.39 -fold) and glycogen synthase 2 (-2.39-fold) are downregulated in muscle of ApOE3*Leiden mice. Unfortunately, $25 \%$ of the genes in the Mus musculus insulin signaling pathway can either not be detected with the Affymetrix mouse chip used or are filtered out because of their low expression. These genes are represented as gray boxes in the insulin pathway (see Fig. 3). 


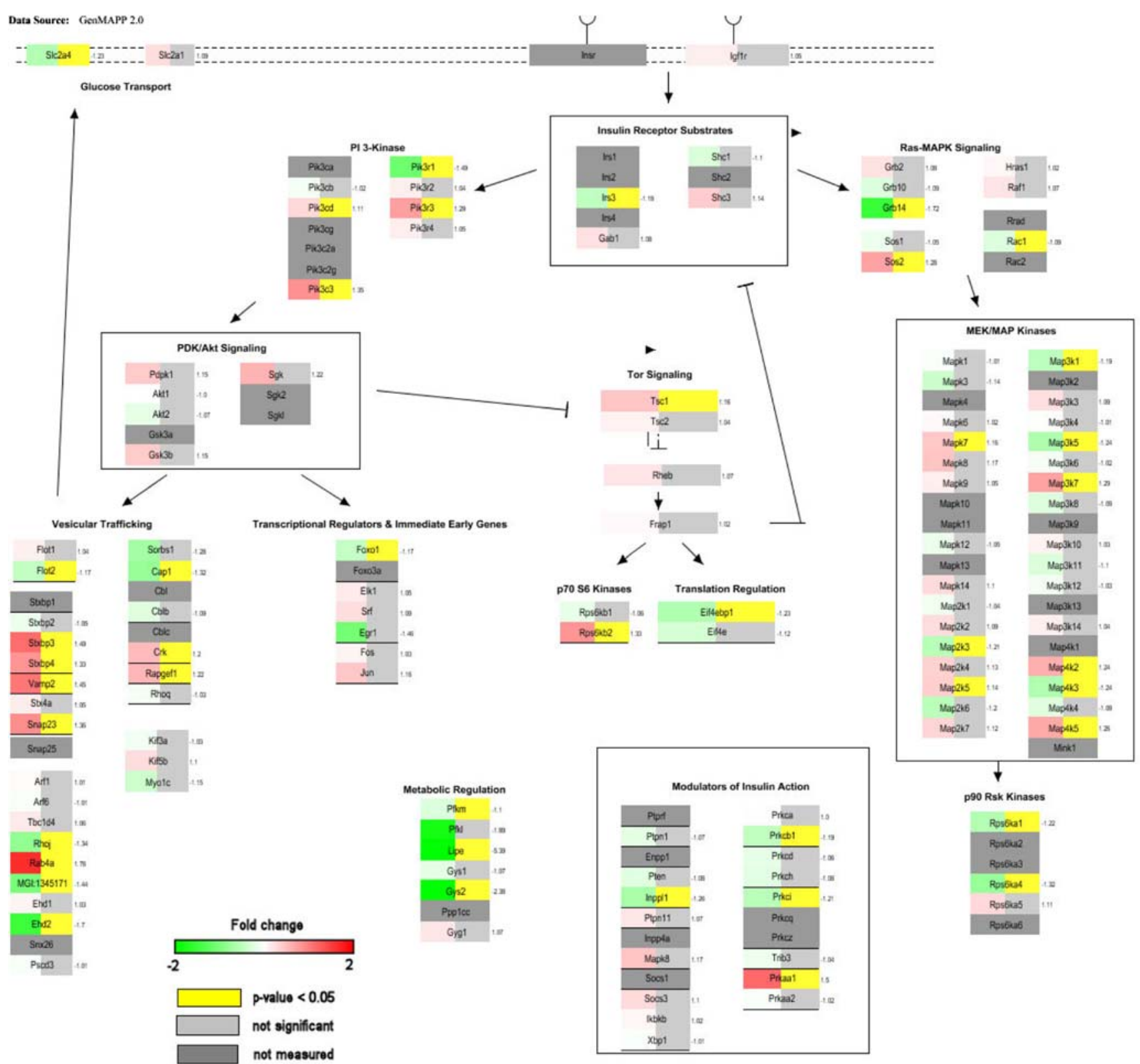

Fig. 3 Gene expression data mapped on the mouse insulin signaling pathway. Fold changes are represented as a gradient and visualized per genebox on the left. Yellow boxes indicate a significance of

$P \leq 0.05$ and are visualized per genebox on the right. Light gray boxes indicate not significant and are also visualized on the right. The dark gray boxes represent the genes that could not be measured

\section{Discussion}

First of all the present study demonstrated that with pathway analysis, using PathVisio, a large variety of processes can be investigated. The significantly regulated genes in muscle of ApOE $3 *$ Leiden mice after 12 weeks of $\mathrm{HF}$ feeding were involved in several biological pathways including fatty acid beta oxidation, fatty acid biosynthesis, insulin signaling, oxidative stress and inflammation. In muscle, it has been demonstrated that increased lipid deposition is associated with the development of insulin

resistance [10]. Therefore, the focus of pathway analysis was in particular on fatty acid metabolism and the insulin signaling. We showed that processes involved in fatty acid metabolism in muscle of ApOE3*Leiden mice are downregulated after 12 weeks of HF feeding, which is in agreement with markedly dropped plasma ketone bodies, a measure of fatty acid beta oxidation (data not shown). Moreover, insulin signaling is regulated after 12 weeks of $\mathrm{HF}$ feeding. The affected muscular insulin signaling proved to be in accordance with the presence of HF-induced insulin resistance (data not shown). 
Fatty acid metabolism

Kelley and coworkers showed that fatty acid-induced insulin resistance is associated with a decreased muscular fatty acid oxidation [6]. We also demonstrated that after HF feeding most genes involved in fatty acid beta oxidation are downregulated. In addition, genes involved in fatty acid biosynthesis were downregulated. For example stearoyl-CoA desaturase 1 (Scd1), which is a critical player in fat metabolism in skeletal muscle [5], is significantly decreased by 2.4 -fold after 12 weeks of HF-diet. These data indicate that even when the intake of fatty acid increases the oxidation is not increased, which could be a reason for the accumulation of lipids in muscle. Moreover, fatty acid biosynthesis is most likely inhibited and thus not contributing to the elevated muscular lipid deposits. However, to unravel the exact mechanism additional studies in which protein levels and enzymatic activity are measured should be performed.

\section{Insulin signaling}

Insulin stimulates muscular glucose uptake via vesicular transport of GLUT4 from the intracellular compartment to the plasma membrane. We demonstrated that the expression of GLUT4 is decreased in muscle under insulin resistant conditions. Several studies have also observed a decrease in GLUT4 mRNA in muscle after feeding a high fat diet $[8,18]$. Strikingly, expression of key genes in the vesicular transport machinery was increased in the insulin resistant muscle of ApOE3*Leiden mice. This is most likely to compensate for the loss of GLUT4 expression. For example, Rab4 and syntaxin4, which interact and are involved in insulin-stimulated glucose uptake [11], are significantly up-regulated after 12 weeks of HF feeding. In addition, the expression of VAMP2, which is required for GLUT4 vesicles incorporation into the plasma membrane in response to insulin [14], is increased. Interestingly, genes involved in the metabolic response of insulin are downregulated, i.e. hormone sensitive lipase and glycogen synthase 2 . Since muscle is not sensitive to 12 weeks of $\mathrm{HF}$ feeding, these data show that the metabolic response to insulin is most likely decreased.

Second, the present study demonstrated that $97 \%$ of the genes in the dataset are recognized by PathVisio, whereas only $20 \%$ of these genes are present on the pathways in the PathVisio repository. This reflects the need for pathways covering more genes in the repository of PathVisio. For example, although both fatty acid oxidation and biosynthesis are available the regulation of fatty acid uptake is missing. A new initiative, Wikipathways [13], enables the development of new pathways on a community base. These new pathways will be added to the repository of pathway tools to make them more complete.
Acknowledgments The present study belongs to the proof of principle study 1 (PPS 1) of NuGO (=the European Nutrigenomics Organisation). The PPS1 is part of the NuGO Proof of Principle Study, which consists of a federate package of Nutrigenomics studies related to energy challenge. We would like to thank TNO Quality of Life, The Netherlands, for providing the gene expression data in muscle.

Open Access This article is distributed under the terms of the Creative Commons Attribution Noncommercial License which permits any noncommercial use, distribution, and reproduction in any medium, provided the original author(s) and source are credited.

\section{References}

1. Adriaens ME, Jaillard M, Waagmeester A, Coort SL, Pico AR, Evelo CT (2008) The public road to high-quality curated biological pathways. Drug Discov Today 13:856-862

2. de Groot P, Reiff C, Mayer C, Muller M (2008) NuGO contributions to Genepattern. Genes Nutr

3. Efron B, Tibshirani R (2002) Empirical Bayes methods and false discovery rates for microarrays. Genet Epidemiol 23:70-86

4. Hansen PA, Han DH, Marshall BA, Nolte LA, Chen MM, Mueckler M, Holloszy JO (1998) A high fat diet impairs stimulation of glucose transport in muscle. Functional evaluation of potential mechanisms. J Biol Chem 273:26157-26163

5. Jiang Z, Michal JJ, Tobey DJ, Daniels TF, Rule DC, Macneil MD (2008) Significant associations of stearoyl-CoA desaturase (SCD1) gene with fat deposition and composition in skeletal muscle. Int J Biol Sci 4:345-351

6. Kelley DE, Goodpaster BH (2001) Skeletal muscle triglyceride. An aspect of regional adiposity and insulin resistance. Diabetes Care 24:933-941

7. Kim JY, Nolte LA, Hansen PA, Han DH, Ferguson K, Thompson PA, Holloszy JO (2000) High-fat diet-induced muscle insulin resistance: relationship to visceral fat mass. Am J Physiol Regul Integr Comp Physiol 279:R2057-R2065

8. Kim Y, Tamura T, Iwashita S, Tokuyama K, Suzuki M (1994) Effect of high-fat diet on gene expression of GLUT4 and insulin receptor in soleus muscle. Biochem Biophys Res Commun 202:519-526

9. Kleemann R, Verschuren L, van Erk MJ, Nikolsky Y, Cnubben NH, Verheij ER, Smilde AK, Hendriks HF, Zadelaar S, Smith GJ, Kaznacheev V, Nikolskaya T, Melnikov A, Hurt-Camejo E, van der Greef J, van Ommen B, Kooistra T (2007) Atherosclerosis and liver inflammation induced by increased dietary cholesterol intake: a combined transcriptomics and metabolomics analysis. Genome Biol 8:R200

10. Kraegen EW, Cooney GJ (2008) Free fatty acids and skeletal muscle insulin resistance. Curr Opin Lipidol 19:235-241

11. Li L, Omata W, Kojima I, Shibata H (2001) Direct interaction of Rab4 with syntaxin 4. J Biol Chem 276:5265-5273

12. Muurling M, Mensink RP, Pijl H, Romijn JA, Havekes LM, Voshol PJ (2003) A fish oil diet does not reverse insulin resistance despite decreased adipose tissue TNF-alpha protein concentration in ApoE-3*Leiden mice. J Nutr 133:3350-3355

13. Pico AR, Kelder T, van Iersel MP, Hanspers K, Conklin BR, Evelo C (2008) WikiPathways: pathway editing for the people. PLoS Biol 6:e184

14. Randhawa VK, Bilan PJ, Khayat ZA, Daneman N, Liu Z, Ramlal T, Volchuk A, Peng XR, Coppola T, Regazzi R, Trimble WS, Klip A (2000) VAMP2, but not VAMP3/cellubrevin, mediates 
insulin-dependent incorporation of GLUT4 into the plasma membrane of L6 myoblasts. Mol Biol Cell 11:2403-2417

15. Ryder JW, Chibalin AV, Zierath JR (2001) Intracellular mechanisms underlying increases in glucose uptake in response to insulin or exercise in skeletal muscle. Acta Physiol Scand 171:249-257

16. Smyth GK (2004) Linear models and empirical Bayes methods for assessing differential expression in microarray experiments. Stat Appl Genet Mol Biol 3(1)

17. van Iersel MP, Kelder T, Pico AR, Hanspers K, Coort S, Conklin BR, Evelo C (2008) Presenting and exploring biological pathways with PathVisio. BMC Bioinformatics 9:399
18. Vettor R, Fabris R, Serra R, Lombardi AM, Tonello C, Granzotto M, Marzolo MO, Carruba MO, Ricquier D, Federspil G, Nisoli E (2002) Changes in FAT/CD36, UCP2, UCP3 and GLUT4 gene expression during lipid infusion in rat skeletal and heart muscle. Int J Obes Relat Metab Disord 26:838-847

19. Zadelaar S, Kleemann R, Verschuren L, de Vries-Van der Weij J, van der Hoorn J, Princen HM, Kooistra T (2007) Mouse models for atherosclerosis and pharmaceutical modifiers. Arterioscler Thromb Vasc Biol 27:1706-1721 